\title{
UAV-BASED SORGHUM GROWTH MONITORING: A COMPARATIVE ANALYSIS OF LIDAR AND PHOTOGRAMMETRY
}

\author{
M. Maimaitijiang ${ }^{1,2}$, V. Sagan ${ }^{1,2, *}$, H. Erkbol ${ }^{1,2}$, J. Adrian ${ }^{1,2}$, M. Newcomb ${ }^{3}$, D. LeBauer ${ }^{4}$, D. Pauli ${ }^{5}$, N. Shakoor ${ }^{6}$, T. C. Mockler ${ }^{6}$ \\ ${ }^{1}$ Geospatial Institute, Saint Louis University, 3694 West Pine Mall, St. Louis, MO 63108, USA \\ ${ }^{2}$ Department of Earth and Atmospheric Sciences, Saint Louis University, St. Louis, MO 63108, USA -(mason.maimaitijiang, \\ vasit.sagan, aishanjiang.yasen, jarrett.adrian) @ slu.edu \\ ${ }^{3}$ United States Forest Service, Intermountain Region, Ogden, UT 84401, USA - maria.newcomb2@usda.gov \\ ${ }^{4}$ Arizona Experiment Station, University of Arizona, Tucson, AZ 85721, USA - dlebauer@email.arizona.edu \\ ${ }^{5}$ School of Plant Sciences, University of Arizona, Tucson, AZ 85721, USA - dukepauli@email.arizona.edu \\ ${ }^{6}$ Donald Danforth Plant Science Center, St. Louis, MO 63132, USA - (nshakoor, tmockler) @ danforthcenter.org
}

\section{Commission III, WG III/10}

KEY WORDS: Unmanned Aerial Vehicle (UAV), LiDAR, photogrammetry, canopy height, leaf area index (LAI), phenotyping

\begin{abstract}
:
Canopy height $(\mathrm{CH})$ and leaf area index (LAI) provide key information about crop growth and productivity. A rapid and accurate retrieval of $\mathrm{CH}$ and LAI is critical for a variety of agricultural applications. LiDAR and RGB photogrammetry have been increasingly used in plant phenotyping in recent years thanks to the developments in Unmanned Aerial Vehicle (UAV) and sensor technology. The goal of this study is to investigate the potential of UAV LiDAR and RGB photogrammetry in estimating crop CH and LAI. To this end, a high resolution 32 channel LiDAR and RGB cameras mounted on DJI Matrice 600 Pro UAV were employed to collect data at sorghum fields near Maricopa, Arizona, USA. A series of canopy structure metrics were extracted using LiDAR and RGB photogrammetry-based point clouds. Random Forest Regression (RFR) models were established based on the UAV-LiDAR and photogrammetry-derived metrics and field-measured LAI. The results show that both UAV-LiDAR and RGB photogrammetry demonstrated promising accuracies in $\mathrm{CH}$ extraction and LAI estimation. Overall, UAV-LiDAR yielded superior performance than RGB photogrammetry in both low and high canopy density sorghum fields. In addition, Pearson's correlation coefficient, as well as RFR-based variable importance analysis demonstrated that height-based metrics from both LiDAR and photogrammetric point clouds were more useful than density-based metrics in LAI estimation. This study proved that UAV-based LiDAR and photogrammetry are important tool in sustainable field management and high-throughput phenotyping, but LiDAR is more accurate than RGB photogrammetry due to its greater canopy penetration capability.
\end{abstract}

\section{INTRODUCTION}

Monitoring crop growth and development is of great significance for agricultural studies (Weiss et al., 2020). As important indicators of crop growth, canopy height $(\mathrm{CH})$ and leaf area index (LAI) provide key information about crop overall conditions such as crop health, stress and nutrient status, as well as crop productivity (Gower et al., 1999; Kimm et al., 2020; Ziliani et al., 2018). An accurate retrieval of crop growth parameters such as $\mathrm{CH}$ and LAI with high efficiency and low cost is critical, particularly in precision agriculture and high-throughput field phenotyping. Field-based direct measurement of $\mathrm{CH}$ and LAI is accurate but often labour-intensive, time-consuming and destructive, while remote sensing techniques provide rapid and non-destructive measurements at larger spatial and higher temporal scales, and have been known as alternative approaches (Tao et al., 2020).

With high spatial resolution, which fulfils the requirements for fine-scale applications (i.e., agricultural field or plot scale), as well as high flexibility and controllability in data collection compared to satellite remote sensing, recently emerged Unmanned Aerial Vehicle (UAV) has advanced the applications of remote sensing technologies, especially in precision agriculture and high-throughput field phenotyping (Maimaitijiang et al., 2020b; ten Harkel et al., 2020). Spectral features (i.e., vegetation indices) derived from UAV-integrated multispectral/hyperspectral sensors have been broadly implemented in monitoring crop growth status, such as CH, LAI and biomass estimation (Maimaitijiang et al., 2017; Tao et al., 2020). However, spectral information is often affected by soil background, atmospheric conditions, as well as optical saturation issue in dense vegetation. Point cloud data from either LiDAR or RGB photogrammetry provides detailed three-dimensional (3D) information about the plant canopy, and can more accurately measure properties of dense canopies because they are less sensitive to saturation after canopies close (Jiang et al., 2020; Sankaran et al., 2015). UAV-based RGB photogrammetric point clouds have been used in canopy height extraction, as well as estimating crop LAI and biomass (Li et al., 2020; Maimaitijiang et al., 2019). With the availability of UAV integrated high resolution LiDAR systems, UAV-LiDAR point clouds were also employed in crop growth monitoring, such as canopy height extraction and biomass prediction (ten Harkel et al., 2020). Compared to RGB photogrammetry, LiDAR often provides higher penetration capability into the canopy, but less costefficient, along with its complexity in terms of operation and data processing (Maimaitijiang et al., 2019; White et al., 2016). Studies about UAV-LiDAR applications in crop growth

\footnotetext{
* Corresponding author
} 
monitoring, especially from a comparative perspective with UAV RGB photogrammetry are less attempted.

This research aims to examine the potential of UAV to estimate crop growth indicators $\mathrm{CH}$ and LAI in sorghum plants from a perspective of LiDAR and RGB photogrammetry comparison. Additionally, the contribution of different canopy structure metrics derived from LiDAR and RGB photogrammetry for LAI estimation was analysed within the formwork of Random Forest Regression (RFR) method.

\section{TEST SITE AND DATA}

\subsection{Test site and experimental setup}

The field experiments were conducted at the University of Arizona's Maricopa Agricultural Center of (elevation $360 \mathrm{~m}$, $33.070^{\circ} \mathrm{N}$ and $111.974^{\circ} \mathrm{W}$ ), located in central Arizona in a semiarid region with low annual rainfall and low relative humidity (Figure 1). During the sorghum growing season in 2019 (May August), the average monthly rainfall was $6.8 \mathrm{~mm}$ (range from 0 in June to $14.1 \mathrm{~mm}$ in July). Soils at the site are characterized as clay loam to sandy loam (Casa Grande soil type).

Sorghum were planted in two adjacent fields (north and south field) at different times in summer 2019. The north field was comprised of a grain sorghum diversity panel planted on 21 May 2019 (15 days later than the southern field trial). The sorghum lines in this grain sorghum experiment were comprised of 234 accessions of the Sorghum Association Panel (SAP) planted in two replicates. There were total 518 plots (37 east-west rows each containing 14 plots), not including the buffer plots on the field edges to minimize edge effects. The dimension of each plot is about $1.5 \mathrm{~m} \times 3.5 \mathrm{~m}$, and each plot covered two rows. The south field experiment was comprised of a sweet sorghum bi-parental population planted on 6 May 2019, two replicates of 166 Recombinant Inbred Lines (RILs) and parents of the GRASSL*RIO population were planted in a split-plot experimental design. There are 672 experimental plots separated by 3 rows of 14 border plots for a total of 714 field plots (excluding external buffer plots planted to minimize edge effect). The dimension of each plot is about $1.5 \mathrm{~m} \times 3.5 \mathrm{~m}$. In addition, to reduce edge effects, lateral and end borders planted in 2-row plots on east/west edges and north/south outer ranges.

At the time of UAV and field data collection from June $24^{\text {th }}$ to June $26^{\text {th }}, 2019$, the north field has relatively lower and sparse sorghum canopy (denoted as L-field), while south field almost reached close canopy stage, which has higher and dense sorghum canopy (denoted H-filed).

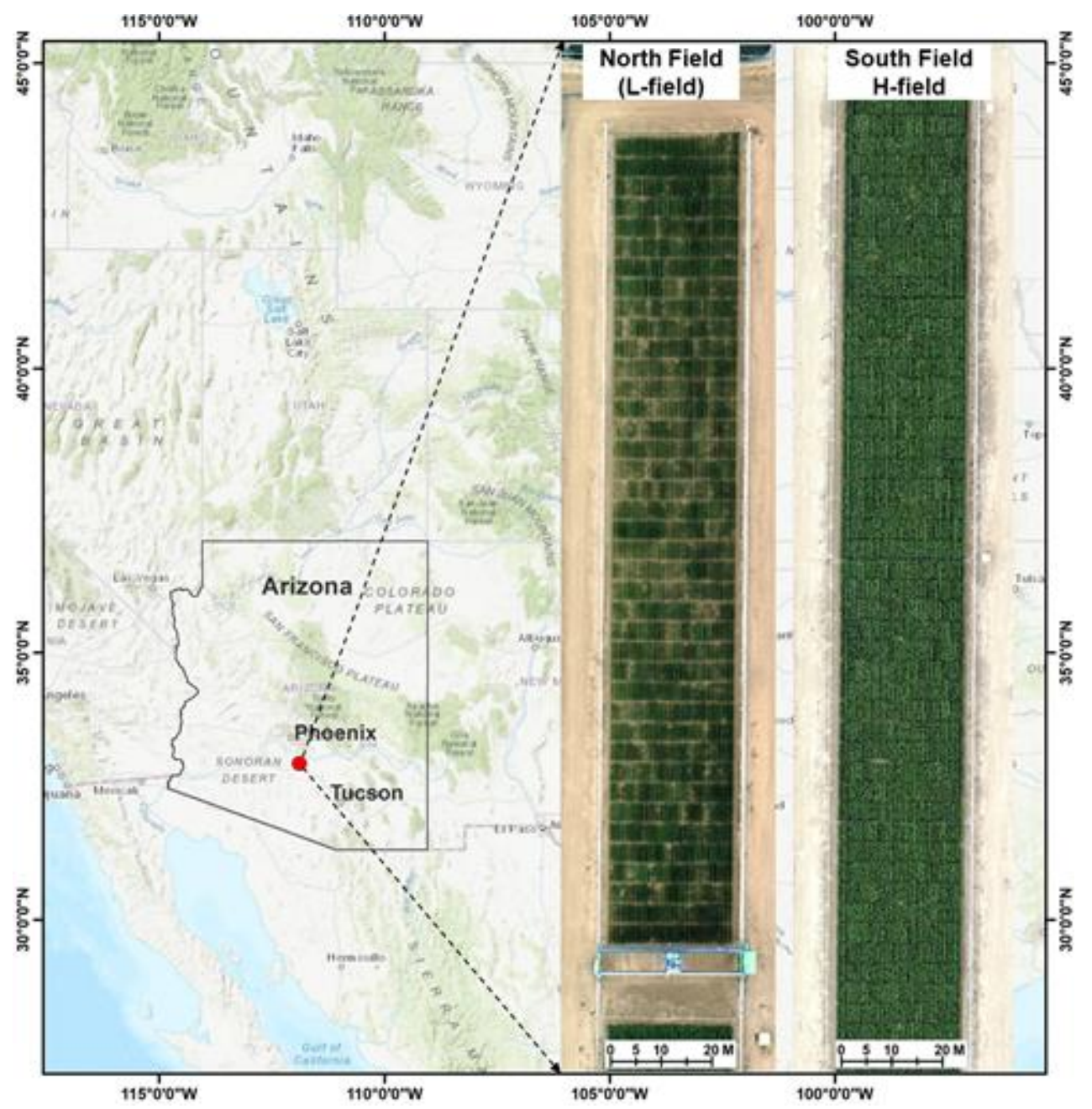

Figure 1. Study site location and UAV-based RGB imagery of two fields (north and south field). The north field was denoted as L-field (Low and sparse canopy), and the south field was denoted as H-field (high and dense canopy). 


\subsection{Data}

2.2.1 Field data collection: to assess the accuracy of $\mathrm{CH}$ derived from UAV LiDAR and photogrammetry point clouds, in situ field measurements of $\mathrm{CH}$ was conducted using a meter stick at 110 locations throughout L-filed, and 92 locations in H-field from June $24^{\text {th }}$ to $26^{\text {th }}, 2019$ (Table 1). CH was measured from the ground vertically to the top of the leaves. It is worth noting that sorghum plots with low to median and high canopy height and density were covered during the measurement to better evaluate the performance of LiDAR and RGB photogrammetry techniques in different canopy conditions.

Non-destructive measurement of LAI was carried out using LAI2200C Plant Canopy Analyzer (LI-COR Inc., Lincoln, NE, USA) from 109 plots in L-field and 87 plots in $\mathrm{H}$-field on June $25^{\text {th }}$ and $26^{\text {th }}$ of 2019. Two above and five below canopy readings of LAI$2200 \mathrm{C}$ were taken along a diagonal transect between two rows within each plot to obtain a LAI value (Maimaitijiang et al., 2017). Field-measured $\mathrm{CH}$ and LAI are summarized in Table 1.

Table 1 Descriptive statistics of field-measured canopy height $(\mathrm{CH})$ and leaf area index (LAI).

\begin{tabular}{cccccccc}
\hline & Field & \multirow{2}{*}{ No. } & Mean & Max. & Min. & SD & $\begin{array}{c}\text { CV } \\
(\%)\end{array}$ \\
\hline \multirow{2}{*}{ Name } & L & 110 & 0.601 & 0.870 & 0.360 & 0.108 & 17.9 \\
$(\mathrm{~m})$ & $\mathrm{H}$ & 92 & 1.21 & 1.58 & 0.910 & 0.128 & 10.6 \\
& $\mathrm{H}+\mathrm{L}$ & 202 & 0.878 & 1.58 & 0.360 & 0.325 & 37.0 \\
\hline \multirow{3}{*}{ LAI } & $\mathrm{L}$ & 109 & 1.30 & 2.93 & 0.420 & 0.491 & 37.8 \\
& $\mathrm{H}$ & 87 & 4.03 & 5.10 & 2.91 & 0.485 & 12.0 \\
& $\mathrm{H}+\mathrm{L}$ & 192 & 2.51 & 5.10 & 0.420 & 1.45 & 57.6 \\
\hline
\end{tabular}

"No. represents the number of samples; SD: standard deviation; CV: coefficient of variation; L-field: lower canopy height density field; H-field: higher canopy height and density field; HL-field: combination of data from $\mathrm{L}$-field and $\mathrm{H}$-field.

2.2.2 UAV data collection: an aerial campaign was conducted to collect LiDAR and RGB data over L-field and H-field separately on June $25^{\text {th }}, 2019$. A DJI Matrice 600 Pro hexacopter (DJI Technology Co. Ltd., Shenzhen, China) (Figure 2a) integrated with a Phoenix Scout-32 system (Phoenix LiDAR Systems, Los Angeles, California, USA) (Figure 2b) was employed to acquire UAV data. The Phoenix Scout-32 system is a lightweight $(2.4 \mathrm{~kg})$ and compact multi-platform (UAV, ground vehicle or backpack) 3D mapping system, and it includes a Velodyne HDL-32 LiDAR sensor and a Sony A7R II RGB camera (Figure 2b). The Velodyne HDL-32 is a 32-channel dualreturn sensor, which has an effective scan rate up to 700,000 $\mathrm{pts} / \mathrm{s}$, field of view (FOV) of $360^{\circ}$ (horizontal) by $41.33^{\circ}$ (vertical), and a reported $\pm 0.02 \mathrm{~m}$ accuracy with an effective range up to $65 \mathrm{~m}$ from the target. Additionally, an UAV onboard GNSS system was integrated to assist point clouds georegistration. Moreover, a ground-based RTK-GPS reference station X900-GNSS (CHCNAV, Shanghai, China) was setup during the aerial data collection to aid flight trajectory correction and improve the accuracy of LiDAR point clouds position. The overlap of LiDAR strips was $75 \%$.

Structure from Motion (SfM) photogrammetry was achieved using the Sony A7R II RGB camera (Figure 2b), which employed a 42-megapixel (MP) CMOS Sensor and 15-mm focal length. UAV flights were conducted at $30 \mathrm{~m}$ AGL with a flight speed of $5 \mathrm{~m} / \mathrm{s}$. The flight mission was planned using Phoenix Flight Planner (www.phoenixlidar.com/flightplan/) and uploaded to the Litchi Mission hub online application (https://flylitchi.com/hub/) for executing the flight mission.
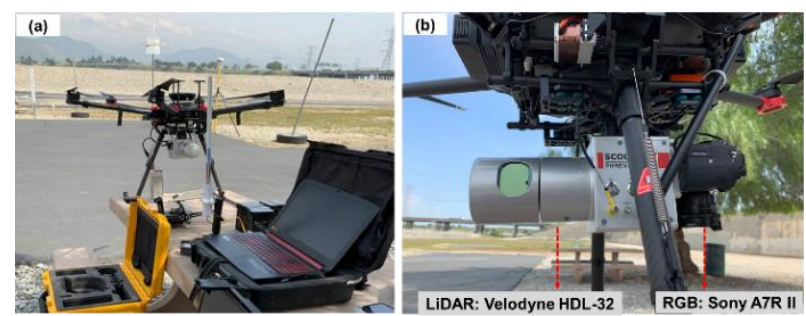

Figure 2. UAV platform and sensors. A DJI Matrice 600 Pro hexacopter integrated with a Phoenix Scout-32 system (a), the Phoenix Scout-32 system includes a Velodyne HDL-32

LiDAR sensor and a Sony A7RII RGB camera (b).

\section{METHODS}

\subsection{UAV data preprocessing}

RGB imagery collections were processed through Pix4Dmapper software (Pix4D SA, Lausanne, Switzerland), which applies orthorectification and mosaicking to generate one whole field image (Figure 1). High density point clouds with las format were generated via Pix4Dmapper's SfM-based photogrammetric module. A number of survey-grade accuracy Ground Control Points were used to produce UAV RGB orthomosaics and point clouds with precise position and scale (Sagan et al., 2019). LiDAR point clouds, flight trajectory files, and GPS data from the ground reference station were loaded into the LiDARMill application (www.phoenixlidar.com/lidarmill, Phoenix LiDAR Systems, Los Angeles, California, USA), which is a cloud -based LiDAR post-processing platform. LiDARMill generates point clouds with las format after applying trajectory correction, noise filtering, and ground/non-ground classification. LiDAR preprocessing involves three steps: combining IMU and GNSS data to generate a smoothed and accurate trajectory, automated flight line detection to reduce processing time by automatically detecting and omitting turns and calibration maneuvers to focus on data-collecting flight lines, and a LiDAR snap process which optimizes alignment parameters and minimizes offsets from multiple flight lines by comparing geometric observations made across overlapping flight lines.

\subsection{Point cloud-based metrics extraction}

A statistical outlier removal algorithm was applied to remove possible outliers in point clouds from LiDAR and RGB photogrammetry (Jiang et al., 2019). Point clouds of L-filed and $\mathrm{H}$-filed from LiDAR and photogrammetry were split by overlaid plot polygons, respectively, and a number of plot-level 3D pointcloud groups were generated. For each plot-level point-cloud group, the $1^{\text {st }}$ percentile of the cumulative probability distribution of original elevation value was regarded as ground elevation (or Digital Elevation Model (DEM)), and the true height value for each point was achieved by subtracting the ground elevation (DEM) from original point heights. Additionally, points below the $1^{\text {st }}$ percentile of the cumulative probability distribution of original elevation were treated as ground points, while the remaining of the points were regarded as non-ground canopy points (Niu et al., 2019).

A set of plot-level canopy structure metrics that are employed for vegetation parameter estimations in previous studies ( $\mathrm{Li}$ et al., 2017; Maimaitijiang et al., 2019) were computed using LiDAR and photogrammetry canopy points, respectively (Table 2 ). 
Table 2 List of canopy structure metrics derived from UAVLiDAR and RGB photogrammetry-based point cloud.

\begin{tabular}{|c|c|}
\hline Metrics & Descriptions \\
\hline Hmax & Maximum of canopy height \\
\hline Hmin & Minimum of canopy height \\
\hline Hmean & Mean of canopy height \\
\hline Hmed & Median of canopy height \\
\hline Hmode & Mode of canopy height \\
\hline Hsd & Standard deviation of canopy height \\
\hline $\mathrm{Hcv}$ & Coefficient of variation of canopy height \\
\hline Hmad & Hmad $=1.4826 *$ median $(\mid$ height - Hmedian $\mid)$ \\
\hline Haad & Haad $=$ mean $(\mid$ height - Hmean $\mid)$ \\
\hline Hper & $\begin{array}{l}\text { Percentile of canopy height: H10, H20, H30, } \\
\text { H40, H50, H60, H70, H80, H90, H95, H98, } \\
\text { H99 }\end{array}$ \\
\hline Hiqr & $\begin{array}{l}\text { The Interquartile Range (iqr) of canopy height, } \\
\text { Hiqr }=\mathrm{H} 75-\mathrm{H} 25\end{array}$ \\
\hline Hskn & Skewness of canopy height \\
\hline Hkurt & Kurtosis of canopy height \\
\hline Hcrd & $\begin{array}{l}\text { Canopy return density is the proportion of } \\
\text { points above the height quantiles (10th, 30th, } \\
50 \text { th, } 70 \text { th, and 90th) to total number of points: } \\
\text { Hd } 10, \mathrm{Hd} 30, \mathrm{Hd} 50, \mathrm{Hd} 70 \text {, and Hd90 }\end{array}$ \\
\hline Hcrr & $\begin{array}{l}\text { Canopy relief ratio of height: } \\
\text { (Hmean- Hmin)/(Hmax - Hmin) }\end{array}$ \\
\hline Hlii & $\begin{array}{l}\text { Laser intercept index (canopy returns/total } \\
\text { returns), a description of fractional canopy } \\
\text { cover }\end{array}$ \\
\hline $\mathrm{Hcg}$ & The ratio of canopy returns and ground returns \\
\hline
\end{tabular}

\subsection{Modeling methods}

A Random Forest Regression (RFR) model was developed to estimate sorghum LAI. RFR is an ensemble regression method that consists of different trees, that are trained by applying bagging and random variable selection process (Breiman, 2001). In this study, we randomly selected $70 \%$ of input canopy structure metrics and corresponding field-measured LAI, and used these to train the RFR model; the remaining $30 \%$ of data were withheld from training and used to test the model. Canopy structure matrices extracted from LiDAR or RGB photogrammetry point clouds were used as input variables for RFR, to estimate sorghum LAI. A grid-search and k-fold crossvalidation technique was employed to obtain optimal parameters during the model calibration phase. Number of tress for RFR method was set at 500, and the max_features parameter which decides how many features each tree in the RFR considers at each split was determined through the grid search procedure (Maimaitijiang et al., 2020a). Root mean square error (RMSE), relative RMSE (RMSE\%), and the coefficients of determination $\left(\mathrm{R}^{2}\right)$ were used to assess model performance:

$$
\begin{aligned}
& \text { RMSE }=\sqrt{\frac{\sum_{i=1}^{n}\left(y_{i}-\hat{y}_{i}\right)^{2}}{n-1}} \\
& \text { RMSE } \%=\frac{\operatorname{RMSE}}{\overline{\mathrm{y}}} * 100
\end{aligned}
$$

where $\hat{y}_{i}$ and $y_{i}$ are estimated and field-measured sorghum LAI, $\bar{y}$ is the mean value of measured LAI, and $n$ is the number of testing samples.
In addition, a permutation-based variable importance of RFR method was used to identify the most influential canopy structure metrics from LiDAR and RGB photogrammetry in LAI estimation. Permutation-based variable importance is achieved by permuting the values of a variable randomly to evaluate the influence on model performance and accuracy (Strobl et al., 2009). The workflow from data processing, canopy structure matrices extraction to model building and evaluation was demonstrated in Figure 3.

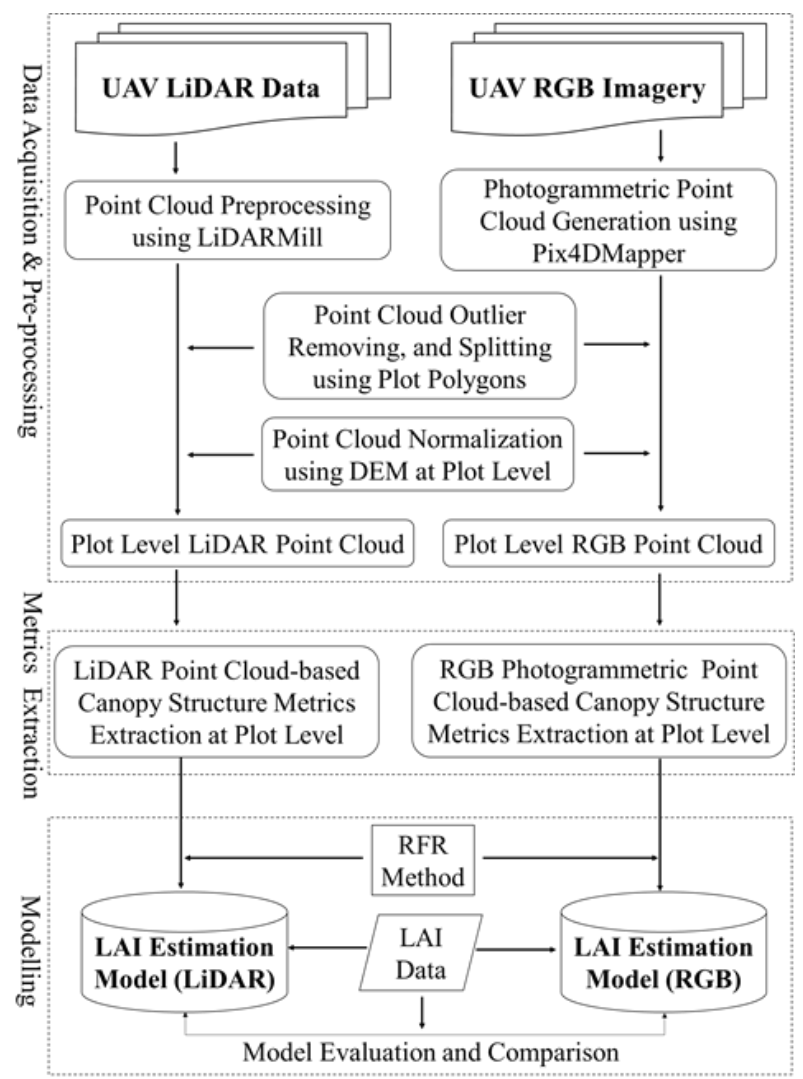

Figure 3. A workflow diagram of data processing, canopy structure matrices extraction and modeling.

\section{RESULTS AND DISCUSSION}

\subsection{LiDAR and Photogrammetry-based CH analysis}

Different percentiles of canopy height (Table 2) were derived from LiDAR and photogrammetry point clouds, and compared to the field measured $\mathrm{CH}$. In this study, H99 (99 percentile $\mathrm{CH}$ ) from both LiDAR and photogrammetry had the highest correlation with measured $\mathrm{CH}$. LiDAR and photogrammetrybased $\mathrm{H} 99$ were plotted against corresponding field measured $\mathrm{CH}$ (Figure 4). Compared to photogrammetry $\mathrm{CH}$, LiDAR yielded higher accuracy with $\mathrm{R}^{2}$ of 0.975 , and RMSE\% of $5.94 \%$, and demonstrated a closer fit toward the 1:1 line. Photogrammetry $\mathrm{CH}$ illustrated a higher spread pattern for both L-field and $\mathrm{H}-$ field, as demonstrated by lower $\mathrm{R}^{2}$ and higher RMSE\%. It is also worth noting that RGB photogrammetry underestimated $\mathrm{CH}$ at both lower and higher values (Figure $4 \mathrm{~b}$ ), which is likely due to the point missing of smaller plants (i.e., L-field) in the point cloud reconstruction process (Maimaitijiang et al., 2019); and the limited penetration of dense canopy (i.e., H-field) inherent to RGB photogrammetry (Figure 7). This led to inaccurate and overestimated DEM, and thus underestimated $\mathrm{CH}$ (White et al., 2016). 

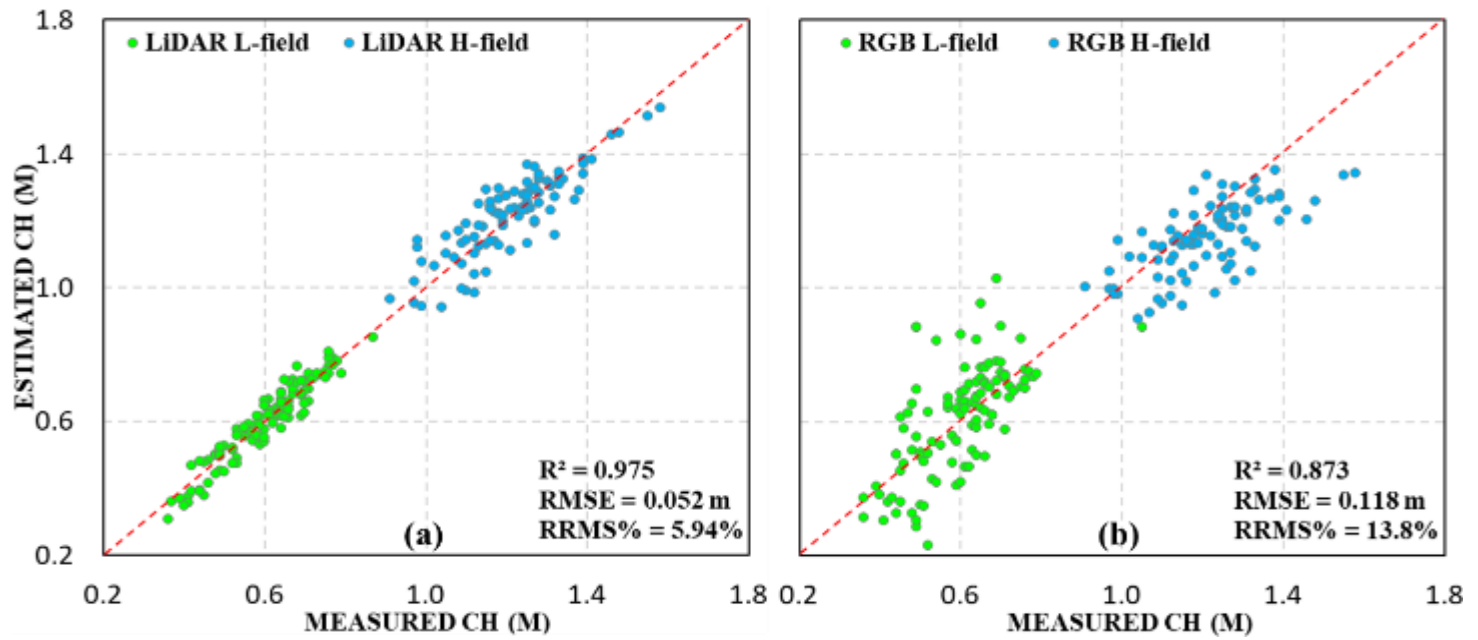

Figure 4. Comparison of field-measured canopy height $(\mathrm{CH})$ with LiDAR and RGB photogrammetry-derived 99 percentiles of canopy height (H99).

To further evaluate the capability of LiDAR and photogrammetry in $\mathrm{CH}$ extraction, plot-level canopy height metrics from both techniques were achieved and compared (Figure 5). Overall, LiDAR and photogrammetry produced consistent and comparable results, as evidenced by the compact distribution pattern of scattered data points around 1:1 line, as well as high $\mathrm{R}^{2}$ values. Nonetheless, given LiDAR-based $\mathrm{CH}$ as a benchmark, an increased underestimation trend was found to photogrammetryderived $\mathrm{CH}$ with higher values (i.e., $\mathrm{CH}>1.0 \mathrm{~m}$ in this case) from H90, H95, H99 to Hmax (Figure 5), and $\mathrm{R}^{2}$ decreased correspondingly from $0.897,0.982,0.878$ to 0.819 . This may be due to the limited canopy penetration capability of photogrammetry method (Figure 7), which often leads to underestimation of $\mathrm{CH}$ at high density (White et al., 2016). For $\mathrm{CH}$ in shorter plots (i.e., $\mathrm{CH}<1.0 \mathrm{~m}$ in this case), H90, H95, H99 and Hmax from LiDAR and photogrammetry are centered around the 1:1 line, and no obvious under or over estimation pattern was noticed. For canopy with a lower height and density (i.e., Lfield), photogrammetry is able to capture vertical information from median/lower canopy and acquire ground points, and produces point clouds with comparable quality to LiDAR (Figure 6 ), and therefore provides more accurate DEM and $\mathrm{CH}$ than photogrammetry in taller and denser plots.
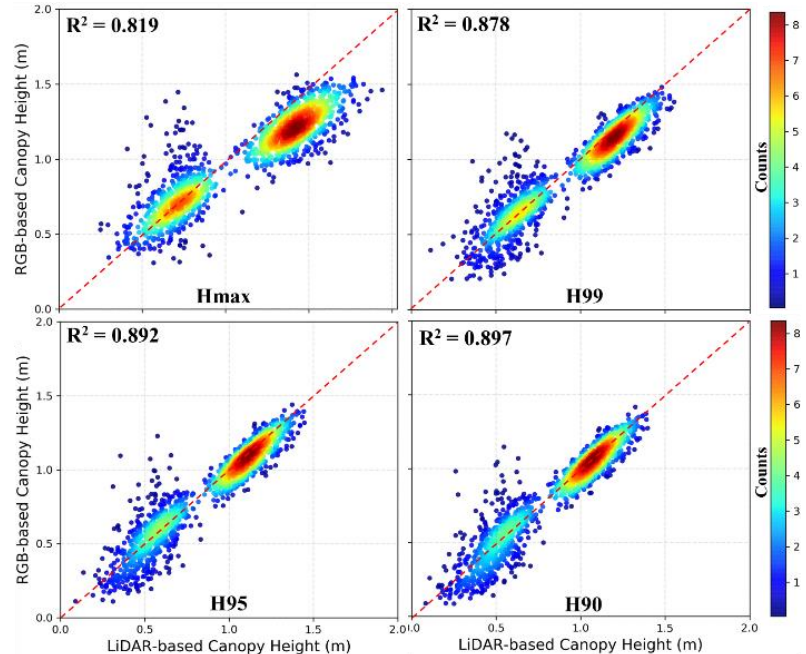

Figure 5. Comparison of LiDAR and RGB photogrammetryderived plot-level canopy percentile heights using density scatter plots. The red dash line is the bisector (1:1 line).
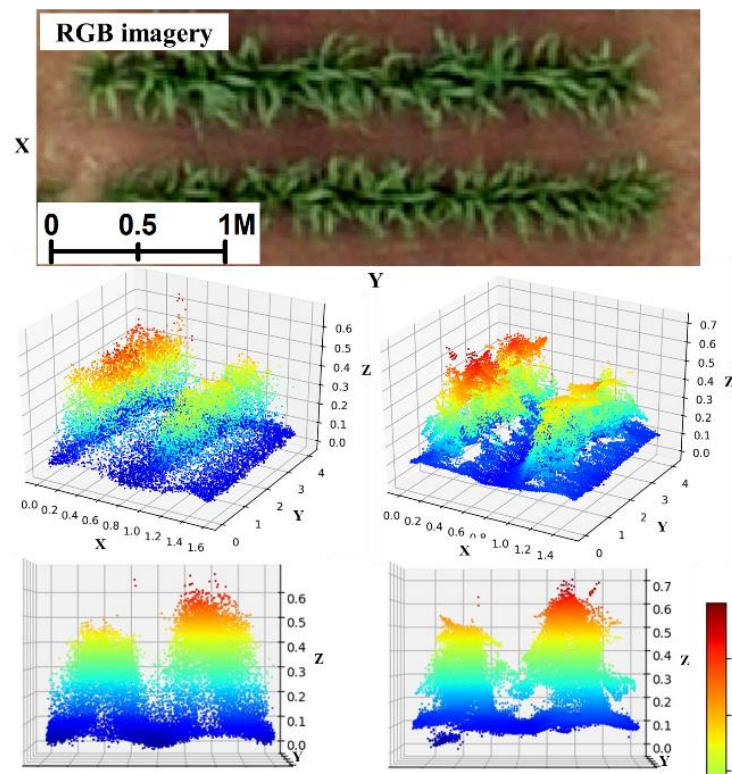

$\begin{array}{lllllllll}1.6 & 1.4 & 1.2 & 1.0 & 0.8 & 0.6 & 0.4 & 0.2 & 0.0\end{array}$
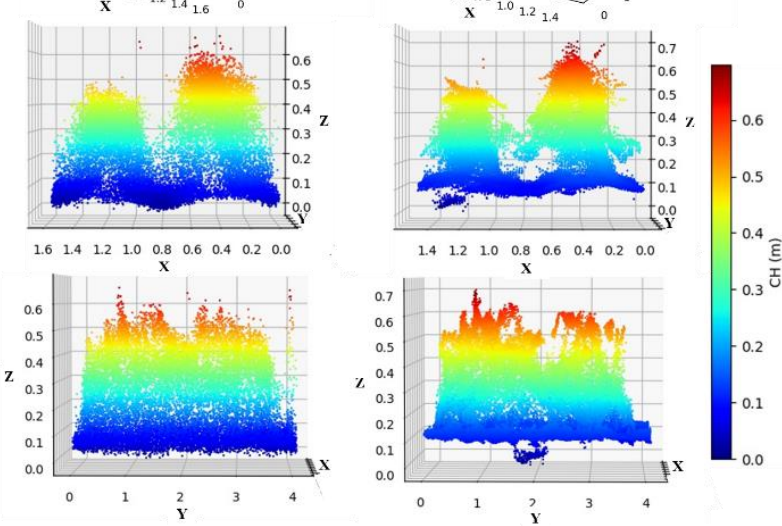

Figure 6. 3D visualization of one selected plot in L-field. (a), (c), (e) are perspective and side views of LiDAR point clouds; (b), (d), (f) are perspective and side views of RGB photogrammetry point clouds. 

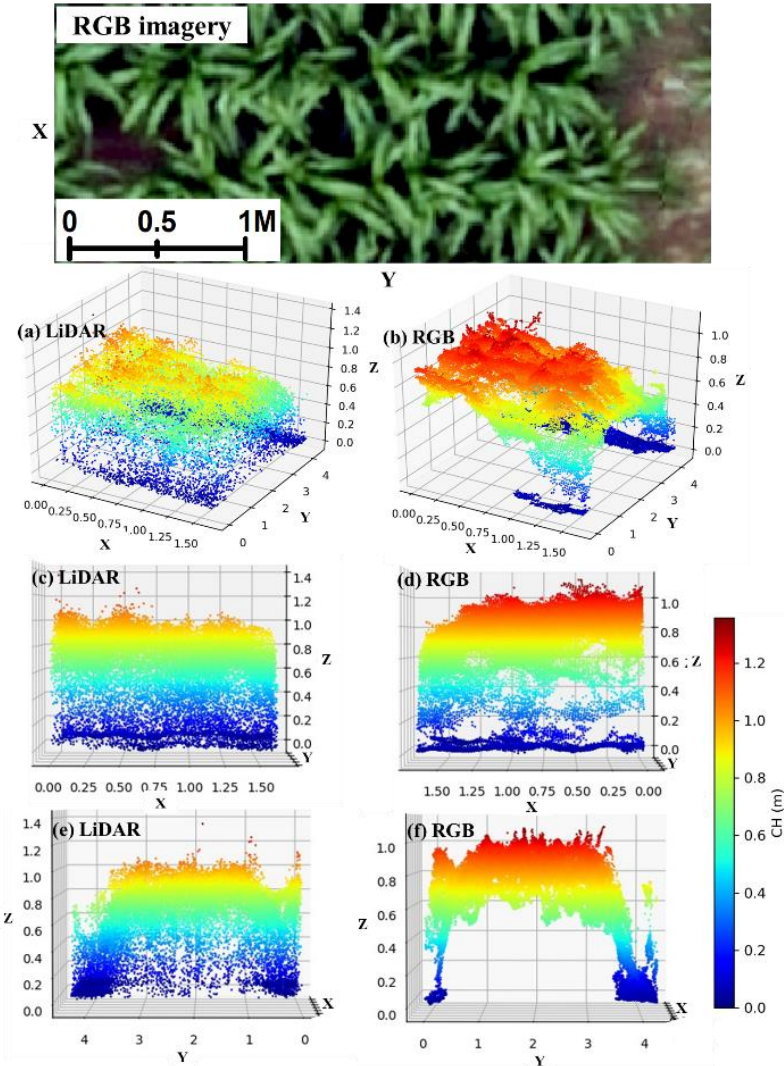

Figure 7. 3D visualization of one selected plot in H-field.

(a), (c), (e) are perspective and side views of LiDAR point clouds; (b), (d), (f) are perspective and side views of RGB photogrammetry point clouds.

\subsection{LiDAR and Photogrammetry-based LAI estimation}

Pearson's correlation analysis was conducted between fieldmeasured LAI data from LH-field and 30 metrics (Table 2) extracted from both LiDAR and photogrammetry to examine the potential of each metric for LAI estimation. Overall, LAI has a relatively strong positive or negative correlation relationship with most of those metrics whether they are computed from point clouds derived from LiDAR or photogrammetry metrics: most metrics have a correlation coefficient $(r)$ higher than 0.8 or lower than -0.8 (Figure 8). In both LiDAR and photogrammetry cases, height-based metrics such as H50, H60, H70, H90 and H99 etc. produced stronger correlation coefficient $(r>0.8)$ than densitybased metrics such as Hd10, Hd30, Hd50, Hd70 and Hd90 $(r<$ 0.4 or $r>-0.4)$. Moreover, the metrics Hmode, H30, Hiqr, Hkurt, Hd10, Hd30 and Hd70 from LiDAR and photogrammetry had opposite correlation relationships (i.e., positive for LiDAR and negative for photogrammetry, or vice versa) with LAI. This is likely due to differences in point clouds from LiDAR and photogrammetry with respect to vertical distribution at different heights, penetration ability, as well as point density.

The RFR method was employed to predict LAI over L-field and $\mathrm{H}$-field separately, as well as using data from both fields combined (LH-field) using the 30 metrics from LiDAR and photogrammetry, respectively. As shown in Table 3, LiDARderived metrics outperformed RGB photogrammetry method in LAI estimation regardless of L-field, $\mathrm{H}$-field, or their combination (LH-field), indicating superior capability of UAVLiDAR in sorghum LAI estimation. While RGB photogrammetry was slightly less accurate, it was comparable to LiDAR in all three cases (Table 3), suggesting RGB photogrammetry has potential as a low-cost technique with sufficiently accurate LAI estimation for some applications.

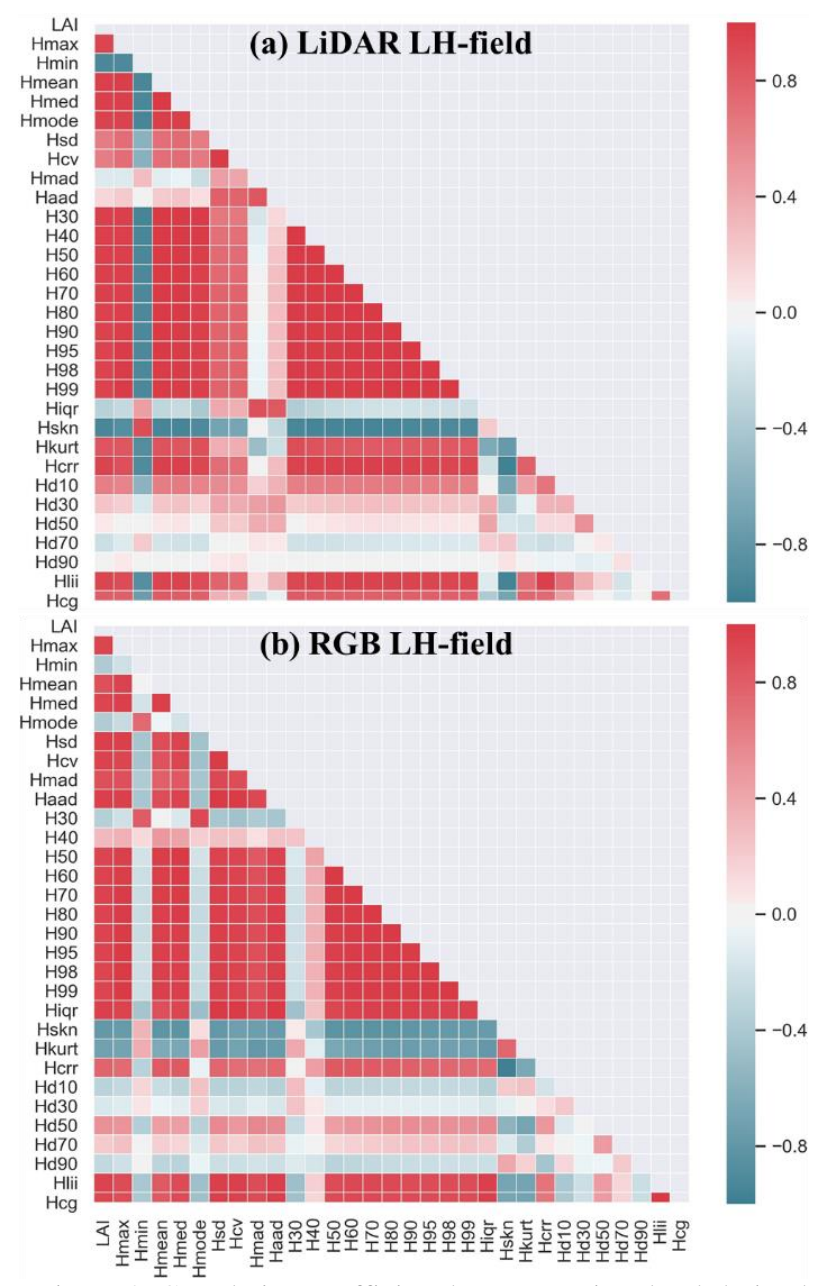

Figure 8. Correlation coefficient between point cloud-derived canopy structure metrics and leaf area index (LAI). LiDARbased metrics for LH-field (a); RGB photogrammetry-based metrics for LH-field.

Additionally, both LiDAR and RGB photogrammetry-derived metrics yielded highest accuracy when combing data from two fields (LH-field) with $\mathrm{R}^{2}$ of 0.950 (LiDAR) and 0.939 (photogrammetry). Combination of LAI data from L-field (lower LAI values) and $\mathrm{H}$-field (higher LAI values) increased the sample size for model building, as well as data range and variations in height, which contributed to the improved accuracy (higher $\mathrm{R}^{2}$ ) of LAI. Furthermore, both LiDAR and photogrammetry techniques generated higher accuracy for Lfield (smaller and sparse sorghum canopy) with $\mathrm{R}^{2}$ of 0.753 (LiDAR) and 0.677 (photogrammetry) than $\mathrm{H}$-field (larger and dense sorghum), which has $\mathrm{R}^{2}$ of 0.416 (LiDAR) and 0.391 (photogrammetry). Limited penetration ability inhibits both LiDAR and photogrammetry in crop applications, although LiDAR suffers less compared to the latter (White et al., 2016), therefore, both techniques are able to capture more internal and vertical canopy structure information from L-field due to the lower and sparser sorghum canopy, which likely led to better prediction of LAI. 
Table 3. Validation statistics of LAI estimation using RFR.

\begin{tabular}{ccccc}
\hline Sensor & VM $^{*}$ & L-field & H-field & LH-field \\
\hline \multirow{3}{*}{ LiDAR } & $\mathrm{R}^{2}$ & 0.753 & 0.416 & 0.950 \\
& RMSE & 0.273 & 0.320 & 0.309 \\
& RMSE\% & 19.2 & 8.10 & 12.2 \\
\hline \multirow{3}{*}{ RGB } & $\mathrm{R}^{2}$ & 0.677 & 0.391 & 0.939 \\
& RMSE & 0.312 & 0.327 & 0.340 \\
& RMSE\% & 22.0 & 8.27 & 13.5 \\
\hline
\end{tabular}

*VM stands for validation metrics for regression models.

The predicted LAI using LiDAR and photogrammetry-derived metrics in the case of L-field, H-field and LH-field were plotted against corresponding field-measured LAI values (Figure 9). As consistent with the high $\mathrm{R}^{2}$, data points are closely distributed around the 1:1 line for the combined LH-field. By contrast, the low $\mathrm{R}^{2}$ is consistent with the spread of data points around the $1: 1$ line in the $\mathrm{H}$-field.

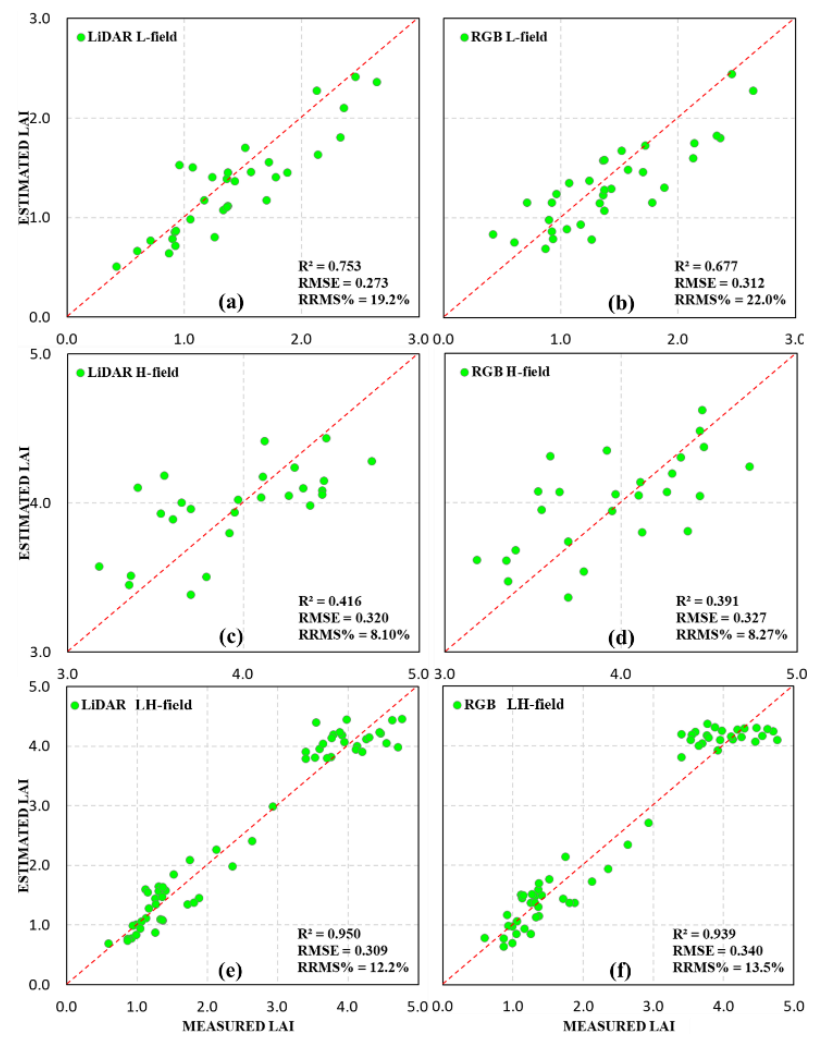

Figure 9. Scatter plots of measured VS. predicted leaf area index (LAI) for L-field using LiDAR (a) and RGB photogrammetry (b), and H-field using LiDAR (c) and RGB photogrammetry (d), as well as LH-field using LiDAR (e) and RGB photogrammetry (f).

\subsection{Importance of point cloud-based metrics}

Figure 10 shows the variable importance ranked in descending order for LAI estimation of LH-field based on RFR model when using LiDAR (Figure 10a) and photogrammetry metrics (Figure 10b), respectively. Overall, variable importance of LiDAR and photogrammetry-based metrics was different in LAI estimation, the most important variable in estimating LAI for LiDAR is Hcg, while it is Hcv for RGB photogrammetry. The least important metric is $\mathrm{Hd} 50$ for both LiDAR and photogrammetry. As evidenced from previous demonstration (Figure 5 to Figure 7) and analysis, point clouds from LiDAR and photogrammetry shown differences in terms of point density, canopy penetration capability, as well as vertical distribution, which are likely due to the different contribution/importance of point cloud-derived metrics from LiDAR and photogrammetry in LAI estimation (Figure 10).

It is worth mentioning that height-based metrics (i.e., H50, H60 and $\mathrm{H} 95$ etc.) yielded higher performance than density-based metrics (i.e., Hd10, Hd30 and Hd50 etc.) in LAI prediction generally. Additionally, although most of the metrics from both LiDAR and RGB photogrammetry demonstrated relatively high correlation with LAI (Figure 8), only a few metrics were identified as influential factors for LAI estimation (Figure 10), this may due to the fact that RFR assesses the importance of each metric jointly by measuring not only the contribution of each variable but in multivariate interactions with other variables as well, and the redundant collinear variables will not be given large importance (Strobl et al., 2009).
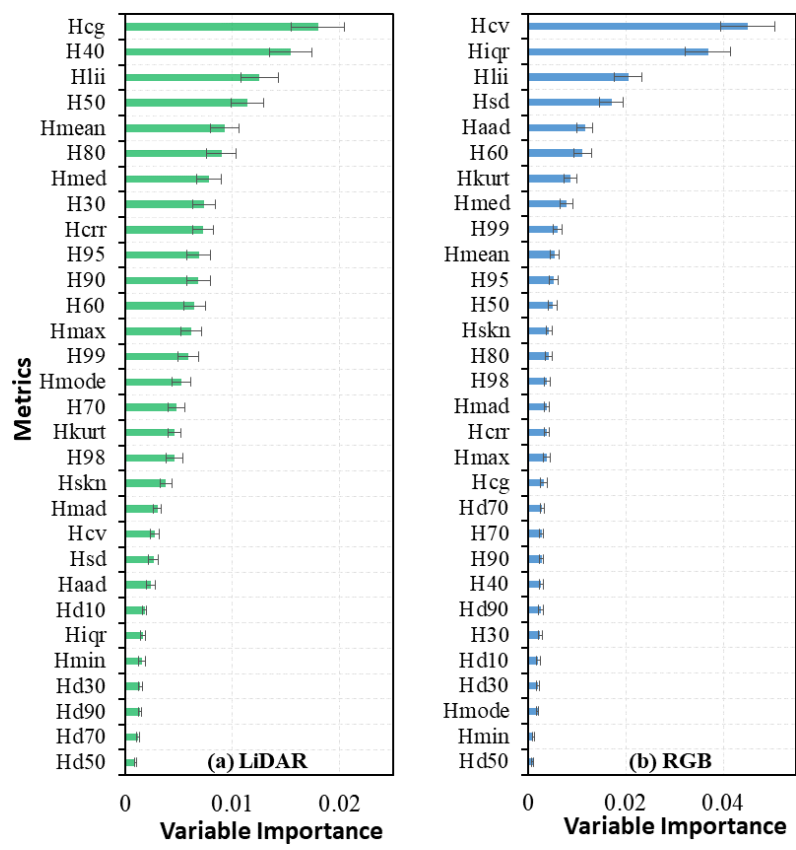

Figure 10. Ranking of height metrics in LAI estimation based on permutation variable importance of RFR. Importance of canopy structure metrics from LiDAR point clouds (a), and RGB photogrammetry point clouds (b). A variable with a higher importance score indicates a larger contribution in LAI estimation.

\section{CONCLUSION}

This study presented a comparative analysis of UAV-based LiDAR and RGB photogrammetry in sorghum growth monitoring, specifically in sorghum canopy height $(\mathrm{CH})$ extraction and LAI estimation. Overall, both UAV-LiDAR and RGB photogrammetry exhibited promising capability in crop growth monitoring by providing accurate and rapid estimations of $\mathrm{CH}$ and LAI in sorghum plants. Nonetheless, UAV-LiDAR presented superior performance over RGB photogrammetry not only in the case of smaller and sparse sorghum canopy (L-field), but for larger and dense sorghum canopy (H-field) as well. In addition, both LiDAR and RGB photogrammetry yielded higher accuracies in LAI estimation for smaller and sparse sorghum (Lfield) than that for larger and dense sorghum (H-field).

Both techniques demonstrated great potential for crop growth monitoring in sparse canopies, however, the relatively poorer performance in dense canopies places some limits on their 
application. For the future work, it will be of interest to evaluate and compare the potential of point clouds from UAV-LiDAR and RGB photogrammetry for other types of plant traits estimation, such as crop aboveground biomass etc. In addition, the potential of both techniques should be tested across different development stages of crops, as well as a variety of crop types. Moreover, the investigation of both techniques in crop growth monitoring could be conducted using other machine learning methods, particularly the state of the art deep learning algorithms.

\section{ACKNOWLEDGEMENTS}

This work was supported by the Department of Energy (ARPAE awards \#DE-AR0000594). The authors thank Sean Hartling, Sidike Paheding, Ebrahim Babaeian and Jeffrey Demieville for their support during the aerial and field data collection.

\section{REFERENCES}

Breiman, L., 2001: Random forests. Machine learning, 45, 5-32.

Gower, S.T., Kucharik, C.J., Norman, J.M., 1999. Direct and indirect estimation of leaf area index, fAPAR, and net primary production of terrestrial ecosystems. Remote sensing of environment, 70, 29-51.

Jiang, Q., Fang, S., Peng, Y., Gong, Y., Zhu, R., Wu, X., Ma, Y., Duan, B., Liu, J., 2019. UAV-Based Biomass Estimation for Rice-Combining Spectral, TIN-Based Structural and Meteorological Features. Remote Sensing, 11, 890.

Jiang, X., Li, G., Lu, D., Chen, E., Wei, X., 2020. StratificationBased Forest Aboveground Biomass Estimation in a Subtropical Region Using Airborne Lidar Data. Remote Sensing, 12, 1101.

Kimm, H., Guan, K., Jiang, C., Peng, B., Gentry, L.F., Wilkin, S.C., Wang, S., Cai, Y., Bernacchi, C.J., Peng, J., 2020. Deriving high-spatiotemporal-resolution leaf area index for agroecosystems in the US Corn Belt using Planet Labs CubeSat and STAIR fusion data. Remote Sensing of Environment, 239, 111615 .

Li, B., Xu, X., Zhang, L., Han, J., Bian, C., Li, G., Liu, J., Jin, L., 2020. Above-ground biomass estimation and yield prediction in potato by using UAV-based RGB and hyperspectral imaging. ISPRS Journal of Photogrammetry and Remote Sensing,162, 161-172.

Li, W., Niu, Z., Chen, H., Li, D., 2017. Characterizing canopy structural complexity for the estimation of maize LAI based on ALS data and UAV stereo images. International journal of remote sensing, 38, 2106-2116.

Maimaitijiang, M.,Ghulam, A., Sidike, P., Hartling, S.,Maimaitiyiming, M., Peterson, K., Shavers, E., Fishman, J.,Peterson, J., Kadam, S., Burken, J., Fritschi, F.B., 2017. Unmanned Aerial System (UAS)-based phenotyping of soybean using multi-sensor data fusion and extreme learning machine. ISPRS Journal of Photogrammetry and Remote Sensing, 134, 4358.

Maimaitijiang, M., Sagan, V., Sidike, P., Daloye, A.M., Erkbol, H., Fritschi, F.B., 2020a. Crop Monitoring using Satellite/UAV Data Fusion and Machine Learning. Remote Sensing, 12, 1357.
Maimaitijiang, M., Sagan, V., Sidike, P., Hartling, S., Esposito, F., Fritschi, F.B., 2020b. Soybean yield prediction from UAV using multimodal data fusion and deep learning. Remote Sensing of Environment, 237, 111599.

Maimaitijiang, M., Sagan, V., Sidike, P., Maimaitiyiming, M., Hartling, S., Peterson, K.T., Maw, M.J., Shakoor, N., Mockler, T., Fritschi, F.B., 2019. Vegetation Index Weighted Canopy Volume Model (CVMVI) for soybean biomass estimation from Unmanned Aerial System-based RGB imagery. ISPRS Journal of Photogrammetry and Remote Sensing, 151, 27-41.

Niu, Y., Zhang, L., Zhang, H., Han, W., Peng, X., 2019. Estimating above-ground biomass of maize using features derived from UAV-based RGB imagery. Remote Sensing, 11, 1261.

Sagan, V., Maimaitijiang, M., Sidike, P., Maimaitiyiming, M., Erkbol, H., Hartling, S., Peterson, K. T., Peterson, J., Burken, J., and Fritschi, F., 2019: UAV/satellite multiscale data fusion for crop monitoring and early stress detection, Int. Arch. Photogramm. Remote Sens. Spatial Inf. Sci., XLII-2/W13, 715722, doi.org/10.5194/isprs-archives-XLII-2-W13-715-2019.

Sankaran, S., Khot, L.R., Espinoza, C.Z., Jarolmasjed, S., Sathuvalli, V.R., Vandemark, G.J., Miklas, P.N., Carter, A.H., Pumphrey, M.O., Knowles, N.R., 2015. Low-altitude, highresolution aerial imaging systems for row and field crop phenotyping: A review. European Journal of Agronomy, 70, 112123.

Strobl, C., Malley, J., Tutz, G., 2009. An introduction to recursive partitioning: rationale, application, and characteristics of classification and regression trees, bagging, and random forests. Psychological methods, 14, 323.

Tao, H., Feng, H., Xu, L., Miao, M., Long, H., Yue, J., Li, Z., Yang, G., Yang, X., Fan, L., 2020. Estimation of Crop Growth Parameters Using UAV-Based Hyperspectral Remote Sensing Data. Sensors, 20, 1296.

ten Harkel, J., Bartholomeus, H., Kooistra, L., 2020. Biomass and Crop Height Estimation of Different Crops Using UAV-Based Lidar. Remote Sensing, 12, 17.

Weiss, M., Jacob, F., Duveiller, G., 2020. Remote sensing for agricultural applications: A meta-review. Remote Sensing of Environment, 236, 111402.

White, J.C., Coops, N.C., Wulder, M.A., Vastaranta, M., Hilker, T., Tompalski, P., 2016. Remote sensing technologies for enhancing forest inventories: A review. Canadian Journal of Remote Sensing, 42, 619-641.

Ziliani, M., Parkes, S., Hoteit, I., McCabe, M., 2018. Intra-season crop height variability at commercial farm scales using a fixedwing UAV. Remote Sensing, 10, 2007. 